\title{
CONVERGENCE OF FOURIER SERIES IN DISCRETE CROSSED PRODUCTS OF VON NEUMANN ALGEBRAS
}

\author{
RICHARD MERCER
}

\begin{abstract}
The convergence of the generalized Fourier series $\sum \pi(x(g)) u(g)$ is considered in the crossed product of a von Neumann algebra by a discrete group. An example from classical theory shows that this series does not converge in any of the usual topologies. It is proven that this series does converge in a topology introduced by Bures which is well suited to a crossed product situation. As an elementary application, we answer the question: In what topology is an infinite matrix (representing a bounded operator) the sum of its diagonals?
\end{abstract}

Let $\mathfrak{U}$ be a von Neumann algebra on a Hilbert space $\mathfrak{E}, G$ a countable discrete group, and $\alpha$ an action of $G$ on $\mathfrak{A}$ (i.e. $\alpha$ is a homomorphism from $G$ to the automorphism group of $\mathfrak{U})$. Then one can define the cross product $\mathfrak{M}=R(\mathfrak{U}, G, \alpha)$ as the von Neumann algebra on $\mathfrak{R}=l^{2}(G ; \mathfrak{S})$ generated by $\{\pi(a): a \in \mathfrak{A}\}$ and $\{u(g): g \in G\}$, where for $\xi \in \mathfrak{N}, g, h \in G, a \in \mathfrak{A}$,

$$
(\pi(a) \xi)(g)=\alpha_{g}^{-1}(a) \xi(g) \quad(u(g) \xi)(h)=\xi\left(g^{-1} h\right) .
$$

To each $x \in \mathfrak{M}$ one can associate a function $x(g): G \rightarrow \mathfrak{A}$ with the idea that $x$ is represented by $\sum \pi(x(g)) u(g)$. It is well known that $x(g)$ uniquely determines $x$, and this is sufficient for many applications. One may also hope that the series actually converges to $x$ in some topology on $\mathfrak{M}$. The purpose of this note is to clarify some of the recent confusion regarding this convergence. We will show that convergence does not take place in any of the usual topologies on $\mathfrak{M}$, and then show that convargence does occur using the $\mathfrak{A}$-topology of Bures.

As an application in a more elementary setting, we consider the question: In what topology is a matrix the sum of its diagonals?

The following example is due to $\mathrm{S}$. Kawamura.

Let $\mathfrak{A}=\mathbb{C}, G=\mathbb{Z}$, and $\alpha$ the trivial action. Then $\mathfrak{M}$ is the von Neumann algebra on $l^{2}(\mathbb{Z})$ generated by the operators $u(n)$, where $u(n) \xi(m)=\xi(m-n)$ for $\xi \in l^{2}(\mathbb{Z})$.

Let $T$ be the unit circle with Lebesgue measure, and let $F: L^{2}(T) \rightarrow l^{2}(\mathbb{Z})$ be the Fourier transform, normalized to be unitary. Then for each $n, F^{*} u(n) F$ is multiplication by $e^{i n t}$ on $L^{2}(T)$ and so is in $L^{\infty}(T) . F^{*} \cdot F$ is a $W^{*}$-isomorphism from $\mathfrak{M}$ to $F^{*} \mathfrak{M} F$. It follows that $F^{*} \mathfrak{M} F$ is generated by the multiplication operators $e^{i n t}$, hence $F^{*} \mathfrak{M} F=L^{\infty}(T)$. It is easy to check that the series $\sum_{g \in G} \pi(x(g)) u(g)$

Received by the editors May 1, 1984 and, in revised form, July 23, 1984. 1980 Mathematics Subject Classification. Primary 46L10; Secondary 47C15.

Key words and phrases. Discrete crossed product, conditional expectation. 
becomes $\sum_{n=-\infty}^{\infty} x(n) e^{i n t}$ in this situation, where $x(n)$ are the Fourier coefficients of a function $x \in L^{\infty}(T)$. The question here is then: In what topology on $L^{\infty}(T)$ does the Fourier series for $x$ converge to $x$ ? We will now show that convergence does not even occur in the weak operator topology, and therefore not in any stronger topology.

Proposition 1. There exists $x \in C(T)$ such that the Fourier series for $x$ does not converge to $x$ in the weak operator topology on $L^{\infty}(T)$.

Proof. Let $x_{N}=\sum_{n=-N}^{N} x(n) e^{i n t}$ denote the $N$ th partial sum of the Fourier series of $x$. Then each $x_{N}$ belongs to $C(T)$. Therefore $\left|x_{N}(0)\right| \leqslant\left\|x_{N}\right\|_{\infty}$. By [5, p. 102] we know that there is a dense $G_{\delta}$ set in $C(T)$ such that, for every $x$ therein, $\sup \left|x_{N}(0)\right|$ $=\infty$. If we consider $\left\{x_{N}\right\}$ to be a collection of linear functionals $x_{N}: L^{1}(T) \rightarrow \mathbb{C}$ by $x_{N}: g \mapsto \int x_{N} g$, then by the Banach-Steinhaus theorem [5, p. 98] we conclude that $\sup \left|\int x_{N} g\right|=\infty$ for all $g$ in some dense $G_{\delta}$ set in $L^{1}(T)$. Since the weak operator topology on $L^{\infty}(T)$ is identical to the weak* topology on $L^{\infty}(T)$ as a Banach space, the desired conclusion follows immediately.

By reversing the spatial isomorphism of $\mathfrak{M}$ with $L^{\infty}(T)$ induced by $F$, we conclude that $\sum \pi(x(g)) u(g)$ need not converge to $x \in \mathfrak{M}$ in the weak operator topology. Erroneous claims for the convergence of this series have been made in [8, p. 366, 4, p. 284 , and 3, p. 785 ]. We now turn to the problem of establishing convergence in the proper topology.

The notation and calculations of [8] will be useful, so we review them here. For $g \in G$ define $P_{g}: \Re \rightarrow \mathfrak{S}$ by $P_{g} \xi=\xi\left(g^{-1}\right)$. Also define $Q_{g}=P_{g}^{*} P_{g}$. Then

$$
\begin{aligned}
& P_{g} u(h)=P_{g h}, \quad u(h)^{*} P_{g}^{*}=P_{g h}, \quad u(h)^{*} Q_{g} u(h)=Q_{g h}, \\
& P_{g} P_{g}^{*}=1, \quad P_{g} P_{h}^{*}=0 \quad \text { if } h \neq g . \\
& \left\{Q_{g}\right\} \text { is an orthogonal set of projections with } \sum_{g \in G} Q_{g}=1 .
\end{aligned}
$$

$\Re$ may be written as $\oplus_{g \in G} \mathscr{S}_{g}$, where each $\mathscr{S}_{g}$ is a copy of $\mathfrak{S}_{\mathrm{g}}$. Then $P_{g}$ is a partial isometry with support $\mathfrak{S}_{g^{-1}}$ and range $\mathfrak{E}$, and $Q_{g}$ is the projection from $\mathfrak{S}_{\mathfrak{E}}$ to $\mathfrak{S}_{g^{-1}}$.

$$
P_{g} \pi(a) P_{g}^{*}=\alpha_{g}(a), \quad \pi(a)=\sum_{g \in G} P_{g}^{*} \alpha_{g}(a) P_{g}, \quad a \in \mathfrak{A} \text { ( } \sigma \text {-weak convergence). }
$$

Define $E: \mathfrak{B}(\Re) \rightarrow \mathfrak{A}$ by $E(x)=P_{e} x P_{e}^{*}$, and define $x(g)=E\left(x u(g)^{*}\right)$ for $x \in \mathfrak{M}$, $g \in G$. ( $e$ is the identity element of $G$.) Then

$$
E\left(u(g) x u(g)^{*}\right)=\alpha_{g}(E(x)), \quad P_{g} x P_{h}^{*}=\alpha_{g}\left(x\left(g^{-1} h\right)\right)
$$

$\pi: \mathfrak{A} \rightarrow \pi(\mathfrak{A}) \subset \mathfrak{M}$ is a $W^{*}$-isomorphism. $\tilde{E}=\pi \circ E$ is a faithful normal conditional expectation from $\mathfrak{M}$ to $\pi(\mathfrak{U})$. Using the formulas given above, one can show that, for any $y \in \mathfrak{M}, E(y)=\sum_{g \in G} Q_{g} y Q_{g}$, the sum converging $\sigma$-weakly.

Note that although [8] assumed $\mathfrak{A}$ to be abelian, this assumption was not necessary for any of the above calculations.

Now consider the locally convex topology on $\mathfrak{M}$ defined by the pseudonorms $x \mapsto \omega \cdot E\left(x^{*} x\right)^{1 / 2}, \omega \in \mathscr{A}_{*}$. This is essentially the $\mathfrak{A}$-topology of Bures [1, p. 48]. It is the appropriate topology here because it works (Proposition 3 ) and it is based in a 
natural way on the crossed product construction. Because $E$ is faithful, the $\mathscr{U}$-topology is Hausdorff. Recall that by " $\sum_{g \in G} \pi(x(g)) u(g)$ converges in topology $T$ " we mean that the net $\left\{\sum_{g \in F} \pi(x(g)) u(g)\right\}_{F}$ on the directed set of all finite subsets $F$ of $G$ converges in the topology $T$.

LEMMA 2. For any finite set $F \subset G$ and $x \in \mathfrak{M}$,

(i) $E\left(x_{F}^{*} x_{F}\right)=E\left(x_{F}^{*} x\right)=E\left(x^{*} x_{F}\right)=E\left(x^{*}\left(\sum_{g \in F} Q_{g^{-1}}\right) x\right)$,

(ii) $E\left(x_{F} x_{F}^{*}\right)=E\left(x_{F} x^{*}\right)=E\left(x x_{F}^{*}\right)=E\left(x\left(\sum_{g \in F} Q_{g}\right) x^{*}\right)$.

Proof. Let $g, k \in G$. Then we have

$$
\begin{aligned}
Q_{k} \pi(x(g)) u(g) & =Q_{k} \pi \circ E\left(x u(g)^{*}\right) u(g) \\
& =Q_{k}\left(\sum_{l \in G} Q_{l} x u(g)^{*} Q_{l}\right) u(g)=Q_{k} x u(g)^{*} Q_{k} u(g)=Q_{k} x Q_{k g} .
\end{aligned}
$$

Taking adjoints we also have

$$
[\pi(x(g)) u(g)]^{*} Q_{k}=Q_{k g} x^{*} Q_{k} .
$$

To check (i) calculate

$$
\begin{aligned}
x_{F}^{*} x_{F} & =\left(\sum_{h \in F} \pi(x(h)) u(h)\right)^{*}\left(\sum_{k \in F} Q_{k}\right)\left(\sum_{g \in F} \pi(x(g)) u(g)\right) \\
& =\sum_{g, h \in F} \sum_{k \in G} Q_{k h} x^{*} Q_{k} x Q_{k g}, \\
x_{F}^{*} x= & \left(\sum_{h \in F} \pi(x(h)) u(h)\right)^{*}\left(\sum_{k \in G} Q_{k}\right) x=\sum_{h \in F} \sum_{k \in G} Q_{k h} x^{*} Q_{k} x, \\
x^{*} x_{F} & =x^{*}\left(\sum_{k \in G} Q_{k}\right)\left(\sum_{g \in F} \pi(x(g)) k(g)\right)=\sum_{k \in G} x^{*} Q_{k} x Q_{k g} .
\end{aligned}
$$

Therefore

$$
\begin{aligned}
E\left(x_{F}^{*} x_{F}\right) & =P_{e} x_{F}^{*} x_{F} P_{e}^{*}=\sum_{g, h \in F} \sum_{k \in G} P_{e} Q_{k h} x^{*} Q_{k} x Q_{k g} P_{e}^{*} \\
& =\sum_{g \in F} P_{e} x^{*} Q_{g^{-1}} x P_{e}^{*}=E\left(x^{*}\left(\sum_{g \in F} Q_{g^{-1}}\right) x\right),
\end{aligned}
$$

where we have used

$$
P_{e} Q_{k h}=\left\{\begin{array}{ll}
P_{e}, & k=h^{-1}, \\
0, & k \neq h^{-1},
\end{array} \quad Q_{k g} P_{e}^{*}= \begin{cases}P_{e}^{*}, & k=g^{-1}, \\
0, & k \neq g^{-1} .\end{cases}\right.
$$

The proofs that $E\left(x_{F}^{*} x\right)$ and $E\left(x^{*} x_{F}\right)$ give the same result are similar. Likewise, the same type of calculations can be used to show (ii).

Proposition 3. For any $x \in \mathfrak{M}, x_{F}$ converges to $x$ and $x_{F}^{*}$ converges to $x^{*}$ in the $\mathscr{H}$-topology on $\mathfrak{M}$.

Proof. By Lemma 2 we have $E\left(x_{F}^{*} x_{F}\right)=E\left(x^{*}\left(\sum_{g \in F} Q_{g^{-1}}\right) x\right)$ and $E\left(x_{F} x_{F}^{*}\right)=$ $E\left(x\left(\sum_{g \in F} Q_{g}\right) x^{*}\right)$. 
Since the nets $\left(\sum_{g \in F} Q_{g^{-1}}\right)_{F}$ and $\left(\sum_{g \in F} Q_{g}\right)_{F}$ are monotone increasing and converge to the identity $\sigma$-weakly, $E\left(x_{F}^{*} x_{F}\right)$ and $E\left(x_{F} x_{F}^{*}\right)$ are monotone increasing and converge $\sigma$-weakly to $E\left(x^{*} x\right)$ and $E\left(x x^{*}\right)$ respectively. Also by Lemma 2 we have $E\left(x_{F}^{*} x\right)=E\left(x^{*} x_{F}\right)=E\left(x_{F}^{*} x_{F}\right)$ and $E\left(x_{F} x^{*}\right)=E\left(x x_{F}^{*}\right)=E\left(x_{F} x_{F}^{*}\right)$. Therefore $E\left(\left(x-x_{F}\right)^{*}\left(x-x_{F}\right)\right)=E\left(x^{*} x\right)-E\left(x_{F}^{*} x_{F}\right)$ and $E\left(\left(x-x_{F}\right)\left(x-x_{F}\right)^{*}\right)=$ $E\left(x x^{*}\right)-E\left(x_{F} x_{F}^{*}\right)$.

For any $\omega \in \mathfrak{U}_{*}, \omega \circ E\left(\left(x-x_{F}\right)^{*}\left(x-x_{F}\right)\right)=\omega\left(E\left(x^{*} x\right)-E\left(x_{F}^{*} x_{F}\right)\right) \rightarrow 0$, showing that $x_{F}$ converges to $x$ in the $\mathfrak{A}$-topology. Likewise,

$$
\omega \circ E\left(\left(x-x_{F}\right)\left(x^{*}-x_{F}^{*}\right)\right)=\omega\left(E\left(x x^{*}\right)-E\left(x_{F} x_{F}^{*}\right)\right) \rightarrow 0,
$$

showing that $x_{F}^{*}$ converges to $x^{*}$ in the $\mathfrak{A}$-topology.

The following result is well known [7, p. 119], but is easily proven in this setting.

Corollary 4. If $\mathfrak{M}$ is finite then for $x \in \mathfrak{M}, \sum_{g \in G} \pi(x(g)) u(g)$ converges in $L^{2}(\mathfrak{M})$ to $x$.

Proof. Let $\tau$ be a trace for $\mathfrak{M}$. Then $\tau \circ \tilde{E}(x)=\tau\left(\sum_{g \in G} Q_{g} x Q_{g}\right)=\tau\left(\sum_{g \in G} Q_{g} x\right)$ $=\tau(x)$. Since $\tau \in \mathfrak{M}_{*}, \tau \circ \pi \in \mathfrak{A}_{*}$, so $\tau \circ \pi \circ E\left(\left(x-x_{F}\right)^{*}\left(x-x_{F}\right)\right)$ converges to zero. But this expression equals $\tau \circ \tilde{E}\left(\left(x-x_{F}\right)^{*}\left(x-x_{F}\right)\right)=\tau\left(\left(x-x_{F}\right) *\left(x-x_{F}\right)\right)$, showing that $x_{F}$ converges to $x$ in $L^{2}(\mathfrak{M})$.

Now that we know what it means for $\sum_{g \in G} \pi(x(g)) u(g)$ to converge, the formulas [8]

$$
x y(g)=\sum_{h \in G} x(h) \alpha_{h}\left(y\left(h^{-1} g\right)\right) \text { and } \quad x^{*}(g)=\alpha_{g}\left(x\left(g^{-1}\right)^{*}\right)
$$

have their intended meaning, representing the components of Fourier series converging to $x y$ and $x^{*}$, respectively.

For the reader's convenience we also mention the notion of "matrix convergence," which gives an alternative way in which $\{x(g)\}_{g \in G}$ represents $x$.

If $x \in \mathfrak{M}$ and $\xi \in \mathfrak{R}$, then since $\sum_{h \in G} Q_{h}$ converges strongly to the identity, $x \xi=\sum_{h \in G} x Q_{h} \xi$, the sum converging in $\Re$, and likewise $x \xi=\sum_{g, h \in G} Q_{g} x Q_{h} \xi$. Using the definition of $Q_{g}$, we have $x \xi=\sum_{g, h \in G} P_{g}^{*} P_{g} x P_{h}^{*} P_{h} \xi$, and hence $(x \xi)\left(g^{-1}\right)=$ $\sum_{g \in G}\left(P_{g} x P_{h}^{*}\right) \xi\left(h^{-1}\right)$, or $(x \xi)(g)=\sum_{h \in G}\left(P_{g^{-1}}^{*} x P_{h^{-1}}^{*}\right) \xi(h)$.

For each, $g, h \in G$ set $x(g, h)=P_{g^{-1}} x P_{h^{-1}}^{*}=\alpha_{g^{-1}}\left(x\left(g h^{-1}\right)\right) \in \mathfrak{A}$. Then $(x \xi)(g)=$ $\sum_{h \in G} x(g, h) \xi(h)$, the sum converging in $\mathfrak{Q}$ for each $g$. This shows $x$ to be represented by an $\mathfrak{A}$-valued matrix indexed by $G$.

Since $x y \xi(g)=\sum_{k} x y(g, k) \xi(k)$, and also $x y \xi(g)=\sum_{k} \sum_{h} x(g, h) y(h, k) \xi(k)$, we conclude that $x y(g, k)=\sum_{h} x(g, h) y(h, k)$, the sum converging strongly. Therefore

$$
\alpha_{g^{-1}}\left(x y\left(g k^{-1}\right)\right)=\sum_{h} \alpha_{g^{-1}}\left(x\left(g h^{-1}\right)\right) \alpha_{h^{-1}}\left(y\left(h k^{-1}\right)\right)
$$

or

$$
x y\left(g k^{-1}\right)=\sum_{h} x\left(g h^{-1}\right) \alpha_{g h^{-1}} y\left(h k^{-1}\right) \quad \text { or } \quad x y(g)=\sum_{h} x(h) \alpha_{h}\left(y\left(h^{-1} g\right)\right),
$$

showing that this formula converges strongly in $\mathfrak{A}$. 
As an application of the ideas presented thus far, consider the following question. Let $A$ be a bounded operator on a Hilbert space $H$ represented as a matrix $\left(A_{i j}\right)$ with respect to some orthonormal basis. For each integer $K$, define the matrix $A(k)$ by $A(k)_{i j}=A_{i j}$ if $i=j+k, 0$ otherwise. The matrices $A(k)$ then represent the different diagonals of the matrix $A$. In what topology does the sum $\sum_{k=-\infty}^{\infty} A(k)$ converge to $A$ ? The example involving Fourier series given earlier, if interpreted as an example regarding Laurent matrices on $L^{2}(\mathbb{Z})$, shows that convergence does not necessarily occur in the weak operator topology.

Let $\left\{P_{i}\right\}$ be the mutually orthogonal one-dimensional projections on the basis vectors of $H$. Then $E(A)=\sum P_{i} A P_{i}$ is a faithful normal conditional expectation from $B(H)$ to $D$, where $D$ is the algebra of diagonal matrices. An elementary calculation then shows that $E\left(A(k)^{*} A\right)=E\left(A^{*} A(k)\right)=E\left(A(k)^{*} A(k)\right)$, whereas $E(A(k) * A(l))=0$ when $k \neq l$. If $F$ is a finite set of integers, define $A_{F}=\sum_{k \in F} A(k)$. It then follows that $E\left(A_{F}^{*} A\right)=E\left(A^{*} A_{F}\right)=E\left(A_{F}^{*} A_{F}\right)$. Calculation also shows that the $i$ th entry of the diagonal matrix $E\left(A^{*} A\right)$ is $\sum_{m}\left|A_{m i}\right|^{2}$, while the corresponding entry of the diagonal matrix $E\left(A_{F}^{*} A_{F}\right)$ is $\sum_{m \in F+i}\left|A_{m i}\right|^{2}$, where $F+i=\{k+i$ : $k \in F\}$. It is then clear that $\left\{E\left(A_{F}^{*} A_{F}\right)\right\}$ is an increasing net converging to $E\left(A^{*} A\right)$ in the strong topology.

We now are able to imitate the proof of Propositon 3 to show the following:

Proposition 5. The partial sums $\sum_{k \in F} A(k)$ converge to $A$ in the locally convex topology on $B(H)$ defined by the pseudonorms $A \rightarrow \omega \cdot E\left(A^{*} A\right)^{1 / 2}, \omega \in D_{*} \simeq l^{1}$.

The author would like to thank Victor Kaftal, Jon Kraus and Jun Tomiyama for valuable suggestions.

\section{REFERENCES}

1. D. Bures, Abelian subalgebras of von Neumann algebras, Mem. Amer. Math. Soc., No. 110, Providence, R. I.. 1971.

2. J. Dixmier, Von Neumann algebras, North-Holland, Amsterdam, New York, 1981.

3. R. Kallman, $A$ generalization of free action, Duke Math J. 36 (1969), 781-789.

4. G. K. Pedersen, $C^{*}$-algebras and their automorphism groups, Academic Press, New York, 1979.

5. W. Rudin, Real and complex analysis, McGraw-Hill, New York, 1966.

6. S. Stratila, Modular theory in operator algebras, Abacus, Tunbridge Wells, England, 1981.

7. N. Suzuki, Crossed products of rings of operators, Tôhoku Math. J. 11 (1959), 113-124.

8. M. Takesaki, Theory of operator algebras. I, Springer, Berlin-Heidelberg-New York, 1979.

Department of Mathematics and Statistics, Wright State University, Dayton, Ohio 45435 